\title{
Modelagem de Processos de Trabalho e Desenvolvimento de Software: Notação e Ferramenta
}

\author{
André L. N. Campos ${ }^{1}$, Toacy C. Oliveira ${ }^{1}$ \\ ${ }^{1}$ Instituto Alberto Luiz Coimbra de Pós-Graduação em Engenharia (COPPE/UFRJ) \\ Caixa Postal 68.511- CEP 21941-972 - Rio de Janeiro - RJ - Brasil \\ ${ }^{1}$ andrecampos@ufrj.br; ${ }^{1}$ toacyecos.ufrj.br
}

\begin{abstract}
Process modeling are becoming more essential to the activities of acquisition and development of systems. There are a number of possible notations and tools for process modeling, and sometimes it isn't an easy choice. This papers try, through research in a real environment, identify selection criteria and recommend the most appropriate notations and tools for process modeling.
\end{abstract}

Resumo. A modelagem de processos mostra-se cada vez mais essencial para as atividades de aquisição e desenvolvimento de sistemas. Há uma série de possíveis notações e ferramentas para a modelagem de processos, e por vezes torna-se complexo o processo de escolha. Esse trabalho busca, através de pesquisa em um ambiente real, identificar critérios de seleção e recomendar as mais adequadas notações e ferramentas de modelagem de processos.

\section{Introdução}

A modelagem de processos, ao longo da última década, tornou-se um importante mecanismo de compreensão dos comportamentos dinâmicos das organizações. Este mecanismo mostra-se superior às técnicas anteriormente empregadas pelos profissionais de sistemas de informação, e pode aumentar o sucesso em projetos de implementação de tecnologias em ambientes dinâmicos (GREEN e ROSEMANN, 2000). Tanto especialistas da área de engenharia de processos quanto da área de tecnologia da informação se beneficiam de uma melhor compreensão dos processos de uma organização. A modelagem conceitual destes processos facilitam o desenvolvimento de software adequado ao suporte dos mesmos (AGULAR-SAVÉN, 2003).

De fato, a modelagem de processos é essencial para representar sistemas complexos, sendo de grande importância para definir as regras dos sistemas de informação integrados aos processos de negócio. Na verdade, a modelagem de processos é o elemento chave para se obter modelos de qualidade que possam suportar a criação de sistemas de informação efetivos (ORTIZ-HERNÁNDEZ, NIETO-ARIZA, et al., 2007). Bargis (BARGIS, 2008) concorda que os softwares atuais são mais do que meros artefatos. Segundo ele, são um complexo fenômeno tecno-social, e que essa complexidade só pode ser tratada através da modelagem de processos, como a base de um modelo de desenvolvimento de software centrada em processos.

Nesse sentido, mesmo antes da modelagem e pesquisas em processos de desenvolvimento de software propriamente ditos, o presente trabalho busca identificar uma notação para a ampla modelagem de processos, que seja de fácil compreensão por 
humanos, e ainda, que tenha potencial para a geração de informações processáveis por máquinas.

\section{Métodos de modelagem}

Há diversas notações, ou métodos, disponíveis para a modelagem de processos, e tantas outras ferramentas que suportam estas notações. Algumas das ferramentas suportam mais de uma notação (GARTNER GROUP, 2008). O presente trabalho se concentra em quatro notações de modelagem de processos, considerando sua relevância para o tema ou sua consolidação no mercado e na academia. As notações são: ARIS, BPMN, SPEM e IDEF0.

A presença da notação ARIS nesse trabalho se justifica por sua ampla utilização, tendo sido objeto de diversos estudos (ARAUJO, CAPPELLI, et al., 2004) (SCHEER, 2003) (SANTOS JR, ALMEIDA e PIANISSOLLA, 2008) (SOUZA, ANDRADE, et al., 2009). Ainda, pesquisas consecutivas do Gartner Group apontaram essa notação como a líder de mercado (GARTNER GROUP, 2008), o que pode ser observado na Figura 1.

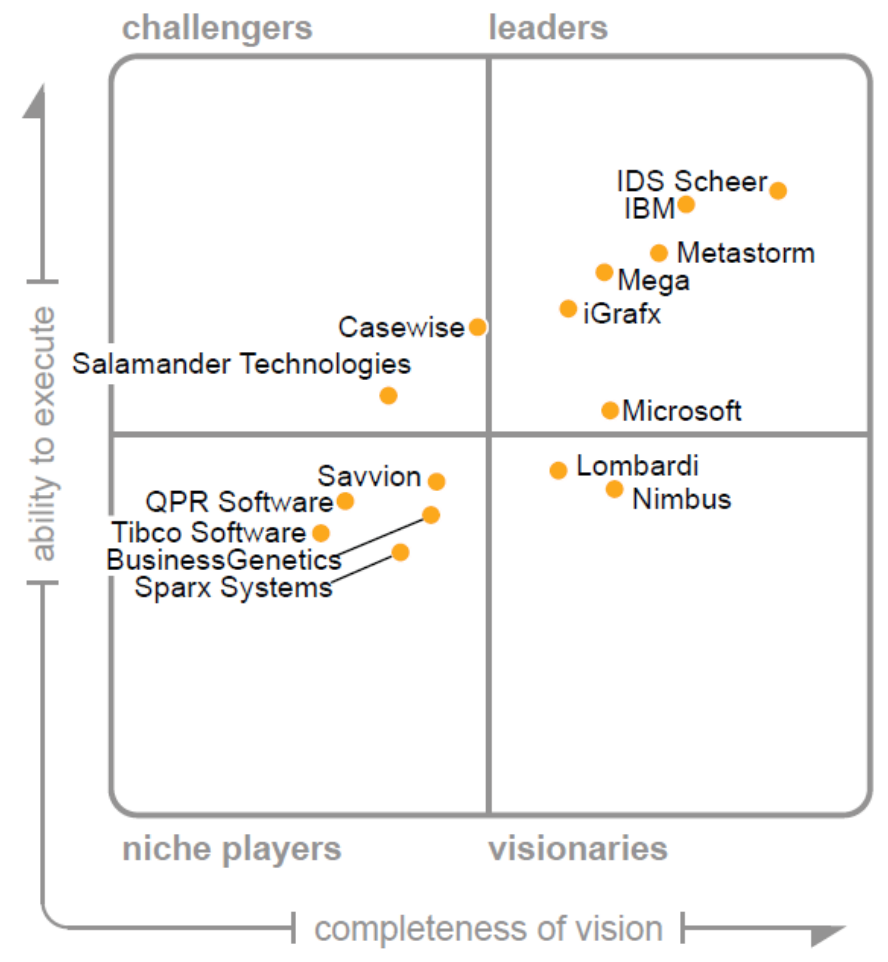

Figura 1. Principais ferramentas de mapeamento de processos (NORTON, BLECHAR e JONES, 2010).

A notação ARIS (ARchitecture for integrated Information Systems) é um framework para modelar a organização. Através desta notação é possível descrever informações da organização e sua arquitetura de integração por meio de seus processos, e diferentes visões organizacionais podem ser descritas. Para a visualização do fluxo de trabalho são utilizados os diagramas de Event-Driven Process Chain (cadeia de processos orientada por eventos), ou simplesmente EPCs. Além da descrição das atividades e seu fluxo, é possível identificar papéis ou perfis, e informações produzidas e consumidas no processo (SANTOS JR, ALMEIDA e GUIZZARDI, 2010). Os principais elementos de um EPC são vistos na Figura 2. 


\begin{tabular}{|c|c|c|}
\hline Tipo & Símbolo & Definição \\
\hline Evento & & $\begin{array}{l}\text { Um Evento descreve uma ocorrência } \\
\text { que causa um efeito (função) }\end{array}$ \\
\hline Função & & $\begin{array}{l}\text { Uma função descreve uma } \\
\text { transformação (uma mudança no } \\
\text { estado do sistema) }\end{array}$ \\
\hline Conectores & $\overbrace{X O R} \bigcap_{A N D}$ & $\begin{array}{l}\text { Um conector estabelece conexöes } \\
\text { lógicas entre eventos e funçöes }\end{array}$ \\
\hline Fluxo & & $\begin{array}{l}\text { Um fluxo descreve uma relação } \\
\text { lógica ou temporal entre funçöes e } \\
\text { eventos }\end{array}$ \\
\hline Caminho & & $\begin{array}{l}\text { Um caminho estabelece uma relação } \\
\text { entre processos. }\end{array}$ \\
\hline
\end{tabular}

Figura 2. Elementos do EPC.

Outra notação considerada é a IDEF0 (Integration Definition 0). Ela foi elaborada a pedido da força aérea americana, na década de 1970, e construída a partir de uma linguagem gráfica bem definida, conhecida como SADT (Structured Analysis and Design Technique). Amplamente utilizada nos Estados Unidos e em alguns países da Europa, a IDEF0 permite representar atividades, fluxos, recursos de suporte, e controles que atuam sobre a atividade. O método é composto basicamente por setas e caixas, sendo a posição da caixa onde a seta se conecta indicativa do seu tipo, ou seja: entrada, saída, controle ou recurso (SOUNG-HIE e KI-JIN, 2000), como pode ser observado na Figura 3.

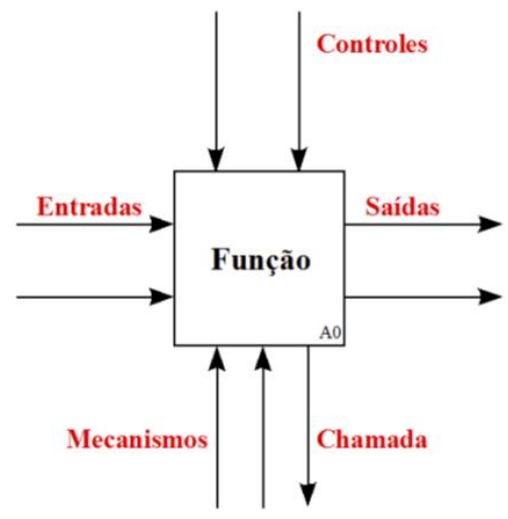

Figura 3. Significado dos elementos do IDEF0.

Um modelo IDEF0 é composto por uma série hierárquica de diagramas que apresentam de maneira progressiva os níveis de detalhes, ampliando o conhecimento sobre o processo e descrevendo funções e suas interfaces no contexto de um sistema. A contribuição do modelador é importante. Ele precisa ter uma visão abstrata do modelo, o que pode ser difícil em grandes sistemas (IEEE, 1998) (SOUNG-HIE e KI-JIN, 2002).

O SPEM (Software \& Systems Process Engineering Metamodel), por sua vez, foi considerado nesse estudo por se tratar de uma notação criada para o domínio do desenvolvimento de software. Foi adotada pela OMG (Object Managent Group) em 2002, e desde então oficializada como um padrão desta organização. Trata-se de um metamodelo que define estereótipos do padrão UML (Unified Modeling Language) para a modelagem de processos de software. De fato, SPEM é uma rede de conceitos e 
relações que se expressa por estereótipos existentes na UML, mas que poderiam ser igualmente descritos por qualquer linguagem suficientemente expressiva, talvez mesmo o inglês, o espanhol ou o português (GONZALES-PEREZ e HENDERSON-SELLERS, 2007). Alguns dos estereótipos podem ser observados na Tabela 1 (ABDALA, LAHOZ e SANT'ANNA, 2003).

Tabela 1. Principais estereótipos do SPEM.

\begin{tabular}{|l|l|l|}
\hline Estereótipo & Comentário & Notação \\
\hline WorkProduct & $\begin{array}{l}\text { Classe de produto de trabalho produzido em um processo e está associado a um } \\
\text { tipo de produto. }\end{array}$ & $\begin{array}{l}\text { Descreve o trabalho executado no processo. Suas subclasses são Activity, } \\
\text { Phase, Iteration e LifeCycle. }\end{array}$ \\
\hline Wuidance & $\begin{array}{l}\text { Informação mais detalhada sobre o elemento associado fornecida aos } \\
\text { praticantes. Exemplos: Guidelines, Metrics, Tools, Checklists e Templates. }\end{array}$ & $\begin{array}{l}\text { Descreve uma parte do trabalho executado por um ProcessRole: as tarefas, } \\
\text { operaçõe e ações que são executadas por um papel. }\end{array}$ \\
\hline Activity & $\begin{array}{l}\text { Define um executante para um conjunto de WorkDefinitions em um processo. } \\
\text { ProcessPerformer }\end{array}$ & $\begin{array}{l}\text { Define responsabilidades sobre WorkProducts específicos e é o executor de } \\
\text { Activities. }\end{array}$ \\
\hline DrocesRole & $\begin{array}{l}\text { Diferentes tipos de WorkProduct como por exemplo Documento Texto, um } \\
\text { Modelo UML, Executável, Biblioteca de Código, etc. }\end{array}$ \\
\hline
\end{tabular}

Outra importante notação é o Business Process Model and Notation (BPMN). O Business Process Management Initiative (BPMI) publicou a especificação da versão 1.0 em maio de 2004, unindo-se a OMG em junho de 2005 com o objetivo de construir um padrão de modelagem de processos que se tornasse a principal referência da área. Atualmente, o BPMN encontra-se em sua versão 2.0 (OMG, 2010).

O modelo BPMN define um diagrama de processos que é baseado em gráficos de fluxo (flowchart) para a descrição de operações de trabalho. Trata-se de uma rede de representações gráficas composta basicamente por atividades (trabalho) e setas (fluxo). O objetivo da notação é ser facilmente compreendida, mas também capaz de gerar informações para ambientes de execução de processos, por exemplo, através de Business Process Execution Languate (BPEL). De fato, faz parte da própria concepção do modelo e estruturação de uma ponte entre os processos de trabalho e a implementação destes processos (WHITE, 2004).

Embora o BPMN possua uma ampla gama de elementos de representação, alguns elementos fundamentais são suficientes para representar a maioria dos processos de trabalho, o que inclui os processos de desenvolvimento de software. Estes elementos são apresentados na Tabela 2.

Tabela 2. Objetos do modelo BPMN.

\begin{tabular}{|l|l|l|}
\hline \multicolumn{1}{|c|}{ Elemento } & \multicolumn{1}{|c|}{ Descrição } \\
\hline Evento & $\begin{array}{l}\text { Um evento é representado por um cícrulo e identifica } \\
\text { quando alguma coisa acontece durante um processo. } \\
\text { Podem ser de início, intermediário ou de fim. }\end{array}$ \\
\hline Atividade & $\begin{array}{l}\text { Uma atividade é representada por um retângulo com } \\
\text { cantos arredondados, e é um termo genérico para } \\
\text { representar trabalho sendo realizado. }\end{array}$ & \\
\hline
\end{tabular}




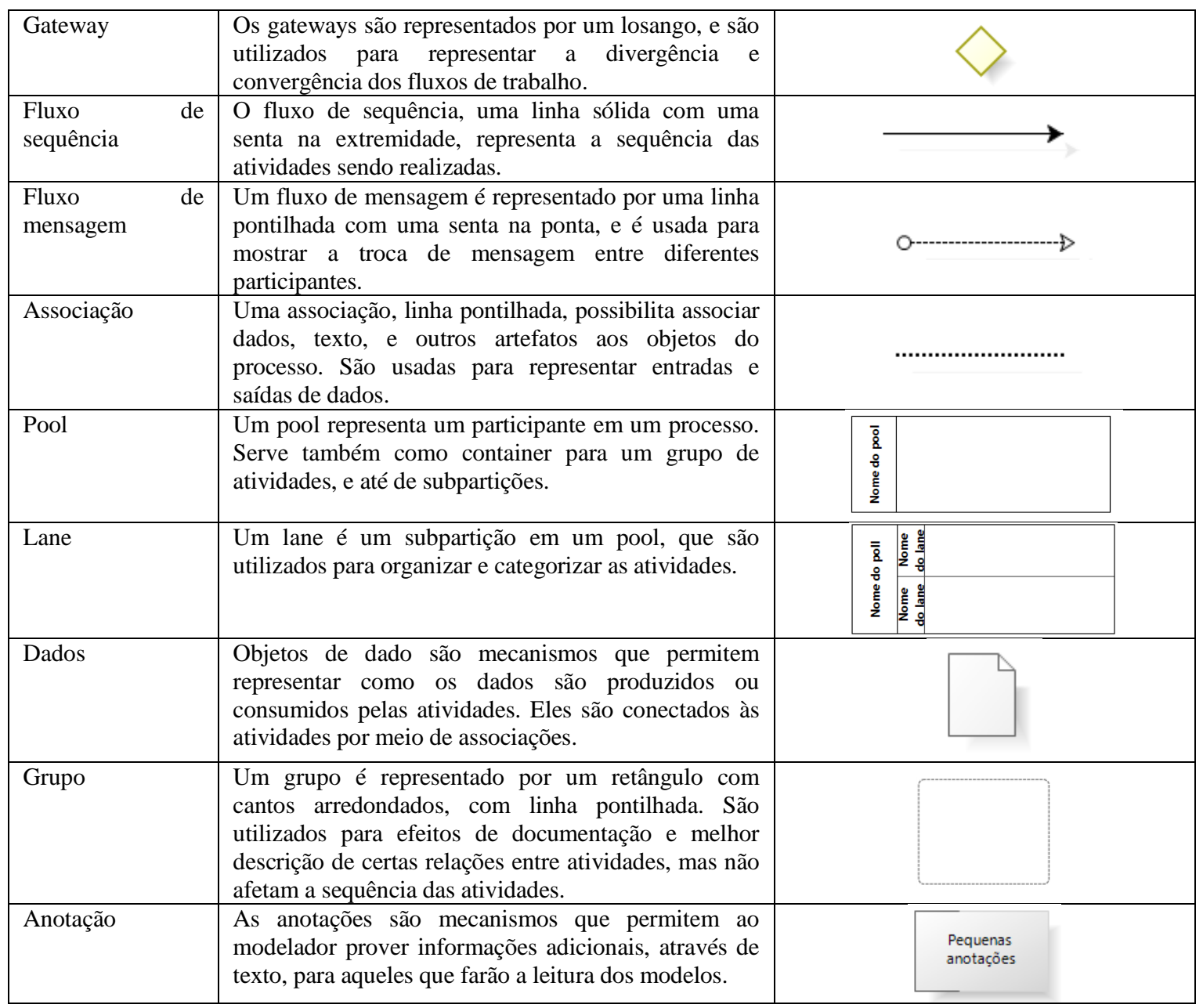

Trabalhos anteriores guardam relação com a presente pesquisa. Por exemplo, Macedo e Schmitz (MACEDO e SCHMITZ, 2001) buscavam encontrar a melhor ferramenta para modelagem de processos de software, mas com vistas a aproximar o desenvolvimento de software dos processos de negócio. Foram avaliadas as ferramentas Aris Toolset e Provision Workbench. Foram considerados aspectos como a arquitetura funcional, método de modelagem de processos, nível de detalhamento dos processos, facilidade de utilização do editor, verificador semântico, entre outros. No entanto, não houve pontuação para estes aspectos e o trabalho não recomenda um ferramenta. Ainda, não foram consideradas as notações em maior profundidade.

Trabalho similar (BENEDICTIS, AMARAL e ROZENDFELD, 2003) realizou pesquisa das notações Aris e IDEF0. Também foram avaliadas ferramentas que suportariam estas notações. $\mathrm{O}$ objetivo da pesquisa seria identificar a notação e ferramenta adequados para a modelagem de processos de trabalho com ênfase no desenvolvimento de produto. Em termos de ferramenta, foram analisadas a Aris Toolset, o Microsoft Visio e o Microsoft Powerpoint. Após a análise das duas notações e três ferramentas, o trabalho conclui fazendo apenas considerações gerais. Há que se considerar ainda que o Microsoft Visio e o Microsoft Poewerpoint são ferramentas de uso genérico e não específicas de modelagem de processos. 


\section{Avaliação das notações}

O objetivo da pesquisa foi identificar uma notação que fosse compreensível por humanos e executável por máquinas. Um trabalho experimental foi conduzido em ambiente real, na Fundação Oswaldo Cruz, uma organização pública da área de Saúde. Este foi realizado em duas etapas: 1) uma análise realizada por profissionais de modelagem de processos; 2) uma análise por pessoas comuns, de diversas áreas da organização.

Com o objetivo de utilizar a mesma referência para análise tanto por profissionais quanto por usuários comuns, optou-se por um processo genérico como exemplo. $\mathrm{O}$ processo hipotético utilizado foi o de emissão de passagem aérea, descrito originalmente em português.

As notações consideradas neste estudo foram avaliadas por quatro profissionais de modelagem de processos. Para a avaliação foi estabelecido um conjunto de critérios, que consolidou propostas de outros trabalhos (MACEDO e SCHMITZ, 2001) (BENEDICTIS, AMARAL e ROZENDFELD, 2003), e adicionou critérios com o objetivo de ampliar a abrangência da pesquisa. Os critérios podem ser observados na Tabela 3. Cada modelador construiu um modelo a partir do processo de referência, utilizando uma notação diferente, ou seja, ARIS, BPMN, SPEM e IDEF0.

Tabela 3. Critérios para análise de modelos pela equipe de modeladores.

\begin{tabular}{|c|c|}
\hline Critério & Detalhamento \\
\hline Possui sintaxe bem definida & $\begin{array}{l}\text { Os objetos se relacionam através de regras que devem ser obedecidas, ou seja, o } \\
\text { método define as ligações corretas entre seus objetos. }\end{array}$ \\
\hline Possui semântica bem definida & $\begin{array}{l}\text { Há objetos específicos que possuem significados distintos, e que não podem ser } \\
\text { utilizados para uma representação diferente daquela para a qual foi criado. }\end{array}$ \\
\hline É bastante difundido & O método é bastante utilizado na academia e/ou no mercado. \\
\hline É de fácil aprendizado & $\begin{array}{l}\text { O método é de fácil aprendizado, podendo ser compreendido sem maiores } \\
\text { conhecimentos e habilidades por parte do usuário. }\end{array}$ \\
\hline É de fácil leitura e compreensão & $\begin{array}{l}\text { Os processos modelados com base no método podem ser compreendidos } \\
\text { facilmente pela maioria das pessoas, mesmo com pouco ou nenhum treinamento } \\
\text { prévio a respeito do método. }\end{array}$ \\
\hline Permite representar atividades & Atividades realizadas por humanos são representáveis com o método. \\
\hline Permite representar informações & Informações, dados ou documentos podem ser representados com o método. \\
\hline Permite representar recursos & $\begin{array}{l}\text { Recursos tais como tecnologias, sistemas, e outros, podem ser representados com } \\
\text { o método. }\end{array}$ \\
\hline $\begin{array}{l}\text { Permite representar integração } \\
\text { entre processos }\end{array}$ & $\begin{array}{l}\text { O acoplamento entre processos, ou seja, a integração entre eles, pode ser } \\
\text { representado com o método. }\end{array}$ \\
\hline $\begin{array}{l}\text { Permite representar os atores que } \\
\text { executam as atividades }\end{array}$ & $\begin{array}{l}\text { Pessoas, cargos ou áreas (departamentos) podem ser representados com o método, } \\
\text { e relacionados à atividade que executam. }\end{array}$ \\
\hline $\begin{array}{l}\text { Permite representar a execução } \\
\text { de uma atividade por mais de um } \\
\text { ator }\end{array}$ & Uma mesma atividade pode ser relacionada com dois executores ao mesmo tempo. \\
\hline $\begin{array}{l}\text { Permite representar um requisito } \\
\text { de sistema }\end{array}$ & $\begin{array}{l}\text { Requisitos de sistema que suportariam uma atividade podem ser representados, ou } \\
\text { ao menos indicados com o método. }\end{array}$ \\
\hline É extensível & O método prevê sua própria extensão pela adição de novos objetos. \\
\hline
\end{tabular}

Após a modelagem dos processos foi realizada uma entrevista com os modeladores onde cada um apresentou o modelo construído, falou das dificuldades e facilidades encontradas e respondeu a questões levantadas pelos demais. Em seguida, cada 
modelador fez a avaliação completa dos quatro modelos considerados, preenchendo a planilha de avaliação.

Esta avaliação foi realizada utilizando-se a escala likert, na qual as respostas para os itens variam de acordo com determinado grau de intensidade, e as categorias são ordenadas igualmente espaçadas e com o mesmo número de categorias em todos os itens (ALEXANDRE, 2003). A avaliação foi feita em cinco níveis, sendo: 1 - Discordo totalmente; 2 - Discordo na maior parte; 3 - Estou em dúvida; 4 - Concordo na maior parte; 5 - Concordo totalmente.

Tabela 4. Avaliação dos modelos de acordo com os critérios definidos.

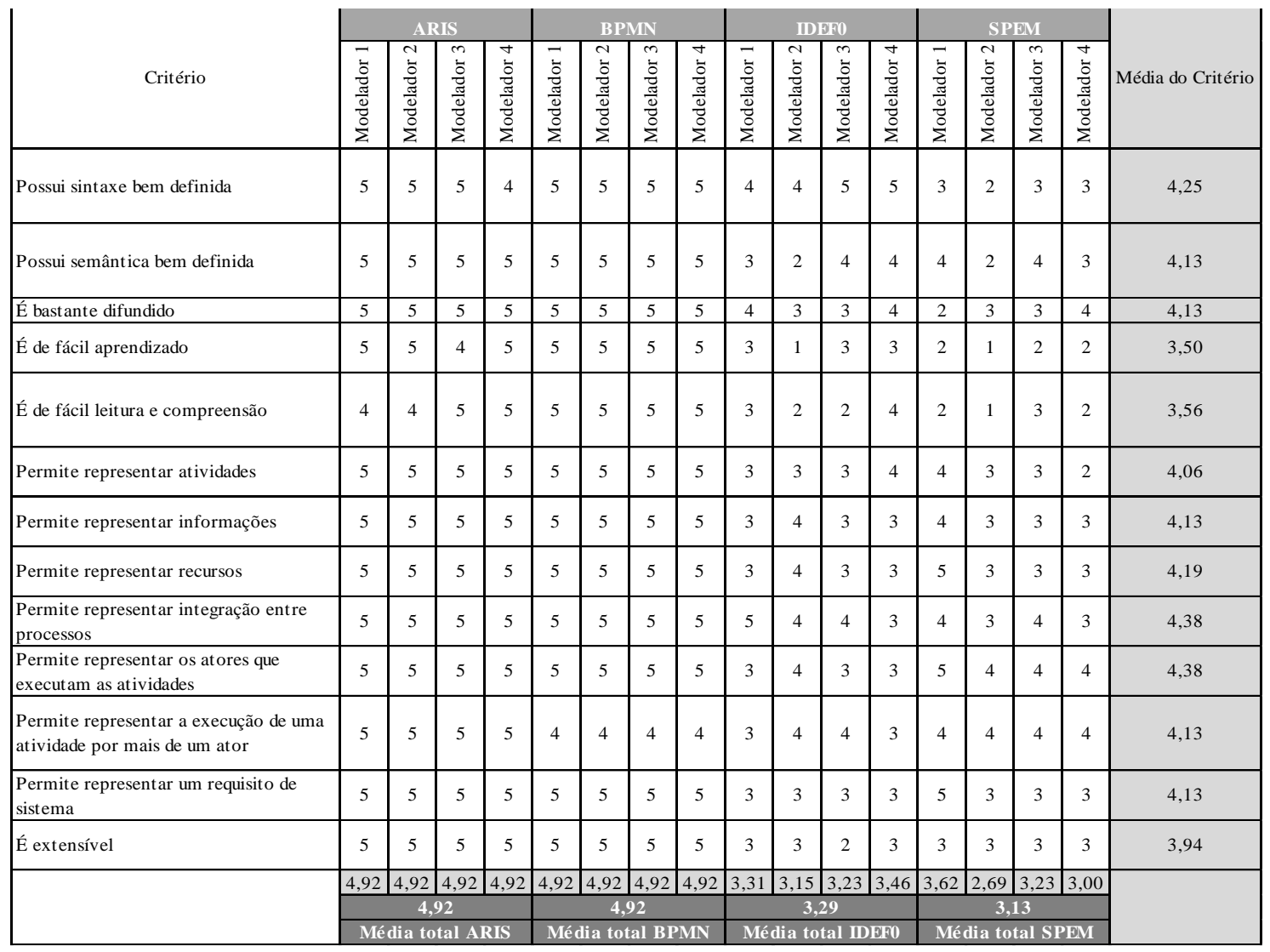

Na Tabela 4 é possível fazer duas observações: quanto cada critério é atendido de maneira geral pelos modelos, e o quanto os modelos estão próximos de atender a todos os critérios. Na análise são calculadas as médias por critério, horizontalmente, as médias por modelador, verticalmente, e as médias totais por ferramenta, que é a média geral daquela determinada notação.

Nesse ponto o trabalho indicava um empate entre as notações ARIS e BPMN, com uma pontuação muito próxima da máxima possível. Os modelos IDEF0 e SPEM ficaram abaixo dos anteriores, e a equipe de modeladores não apresentava tendência para nenhuma das duas mais bem colocadas na avaliação.

Com o objetivo de ampliar a pesquisa e verificar o grau de compreensão dos modelos que ficaram empatados em primeiro lugar, outra pesquisa foi conduzida. Dessa vez, envolvendo 50 pessoas de diversas áreas da organização, sem qualquer formação na área de TI ou de Processos. Participaram secretárias, pessoas da área de RH, Compras e outras, em geral profissionais da área administrativa. 
A pesquisa foi composta do processo de referência modelado tanto em notação ARIS quanto em BPMN, do próprio processo de referência em português, e de um questionário para indicar qual dos dois modelos seria de mais fácil compreensão. Verificou-se uma vantagem da BPMN sobre a ARIS, conforme Figura 4.

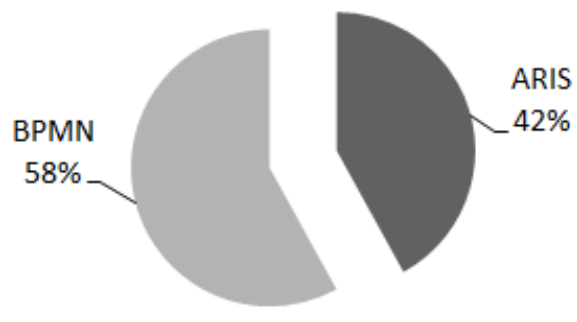

Figura 4. Pesquisa de compreensão de modelos com pessoas de diversas áreas.

A partir dessa etapa a notação BPMN passou a ser considerada a mais adequada em termos de compreensão humana, e as etapas seguintes da pesquisa se concentraram nesta notação, já que o aspecto da compreensão por humanos era uma premissa do trabalho. Ademais, ao contrário da notação ARIS, a notação BPMN é um padrão aberto da OMG e conta com o apoio de importantes atores desse mercado, como IBM, Oracle, SAP, Unisys e a própria IDS Sheer.

\section{Avaliação das ferramentas}

Considerando a vantagem obtida pela notação BMPN, o trabalho seguiu avaliando ferramentas disponíveis no mercado brasileiro que suportassem ao menos essa notação. A fase de análise de ferramentas contou com o apoio de um grupo de seis profissionais de modelagem de processos, incluindo o grupo original de quatro modeladores e mais dois profissionais que se juntaram ao trabalho nesta fase.

Inicialmente foram definidas as características desejáveis, ou seja, os critérios a serem considerados na avaliação das ferramentas. Critérios utilizados em outros trabalhos similares, como (BENEDICTIS, AMARAL e ROZENDFELD, 2003), e (RECKER, 2010), formaram a base dos critérios do presente trabalho. Os critérios são apresentados na Tabela 5.

Tabela 5. Critérios para análise de ferramentas.

\begin{tabular}{|c|l|l|}
\hline $\mathbf{1}$ & Requisitos da Ferramenta & Detalhamento \\
\hline 1.2 & Linguagem no idioma nato (Português - BR) & A ferramenta possui tradução para o Português - BR \\
\hline 1.3 & Possui recurso Colaborativo & $\begin{array}{l}\text { Permite que múltiplos modeladores trabalhem simultaneamente em } \\
\text { um mesmo processo. }\end{array}$ \\
\hline 1.4 & Repositório integrado para os modelos & Permite o compartilhamento de modelos em um repositório único. \\
\hline 1.5 & Permite Versionamento & Faz o controle de versões. \\
\hline 1.6 & Permite Publicação WEB & Permite que os modelos sejam publicados via WEB. \\
\hline 1.7 & $\begin{array}{l}\text { Permite exportação para outras ferramentas através } \\
\text { de XPDL. }\end{array}$ & Exportam dados através do padrão XPDL. \\
\hline 1.8 & $\begin{array}{l}\text { Permite integração com ferramentas de Workflow } \\
\text { via BPEL }\end{array}$ & Integra-se a ferramentas de Workflow através do padrão BPEL \\
\hline 1.9 & Permite validação para o método & Faz a validação do Método observando as regras da notação. \\
\hline
\end{tabular}




\begin{tabular}{|c|l|l|}
1.10 & $\begin{array}{l}\text { Permite consulta ao banco de dados por entidade } \\
\text { ontológica }\end{array}$ & $\begin{array}{l}\text { Permite buscar, através de um objeto, suas relações com outros } \\
\text { objetos do banco (repositório). }\end{array}$ \\
\hline 1.11 & Navegação entre modelos de diferentes níveis & $\begin{array}{l}\text { Possibilita criar links de navegação entre objetos, final de um e } \\
\text { início de outro. }\end{array}$ \\
\hline 1.12 & Atributos adicionais para os objetos do modelo & $\begin{array}{l}\text { Para um objeto permite abrir janela com atributos que podem ser } \\
\text { preenchidos para o objeto em questão }\end{array}$ \\
\hline 1.13 & $\begin{array}{l}\text { Permite a utilização de mais de uma notação de } \\
\text { modelagem }\end{array}$ & $\begin{array}{l}\text { Permite modelar em diferentes notações, como BPMN, ARIS, e } \\
\text { outros. }\end{array}$ \\
\hline 1.14 & Permite criar novos objetos personalizados & $\begin{array}{l}\text { Permite criar um novo símbolo (objeto) a partir de outro, uma } \\
\text { espécie de herança visual. }\end{array}$ \\
\hline 1.16 & $\begin{array}{l}\text { Permite adicionar hyperlinks para outras } \\
\text { documentações }\end{array}$ & $\begin{array}{l}\text { Possibilita apontar para arquivos de apoio, com documentos Word, } \\
\text { planilhas, vídeos, entre outros. }\end{array}$ \\
\hline 2 & Seseja usar & $\begin{array}{l}\text { Havendo muitos objetos, permite criar filtro com apenas os objetos } \\
\text { necessários a determinado trabalho. }\end{array}$ \\
\hline 2.1 & Existem cursos para treinamento & Detalhamento \\
\hline 2.2 & Existem comunidades virtuais de apoio & Possui cursos disponíveis para treinamento da ferramenta. \\
\hline 2.3 & Existem publicações de apoio e suporte disponíveis & Possui comunidades e fóruns de consulta disponíveis na web. \\
\hline
\end{tabular}

O trabalho utilizou o conceito de peso, com o objetivo de equalizar o grau de importância entre diferentes critérios. Com a exceção do critério "Permite integração com ferramentas de workflow via BPEL", que recebeu peso 4, os demais critérios classificados como requisitos da ferramenta receberam peso 2 . Os critérios de suporte adicional receberam peso 1 , por serem menos relevantes.

As ferramentas consideradas foram aquelas encontradas no mercado brasileiro: Mega; Tibco; QPR; QPR Express; BizAgi Free; BizAgi Express; Aris Free; Aris completa. Para as que apresentavam opções gratuitas, essas foram avaliadas independentemente.

Os modeladores foram divididos em dois grupos, sendo cada grupo responsável pela análise de um conjunto de ferramentas. Em seguida, os grupos se reuniram para apresentar e discutir os resultados, gerando uma avaliação de consenso. O resultado da avaliação pode ser visto na Tabela 6. A aplicação dos pesos sobre as notas está identificada nas colunas com o título NAj (nota ajustada).

De acordo com a análise, e levando-se em conta especificamente os critérios de requisitos de ferramenta, a ferramenta ARIS Full (completa e paga) recebeu a maior pontuação média. Considerando exclusivamente os critérios de suporte adicional, as ferramentas QPR, BizAgi Free e BizAgi Express ficaram empatadas na liderança. Na média final, a ferramenta ARIS Full recebeu a maior pontuação.

Observando as médias de atendimento dos critérios pelas ferramentas é possível compreender quais desses critérios os fabricantes estão mais preocupados em atender. Os três critérios mais bem colocados são: 1 - atributos adicionais para os objetos do modelo; 2 - publicação do modelo na WEB; 3 - mecanismo de validação para o modelo.

Outra observação é que a média da pontuação final das ferramentas pagas não é tão distante da média da pontuação final das ferramentas gratuitas. Este resultado pode indicar um fortalecimento das versões gratuitas.

Há outros fatores que podem ser considerados sobre ferramentas. Por exemplo, a ferramenta QPR ficou apenas um décimo atrás da ARIS completa, o que pode recomendar seu uso. Ainda, questões de custo podem recomendar o uso de uma ferramenta gratuita, como o BizAgi gratuito, que obteve pontuação maior até do que ferramentas pagas. 
Tabela 6. Análise das ferramentas de acordo com os critérios.

\begin{tabular}{|c|c|c|c|c|c|c|c|c|c|c|c|c|c|c|c|c|c|c|}
\hline 1 & Requisitos da Ferramenta & PESO & MEGA & $N A j$ & TIBCO & $N A j$ & $Q P R$ & $N A j$ & $\begin{array}{c}\text { QPR } \\
\text { EXPRESS }\end{array}$ & $N A j$ & $\begin{array}{l}\text { BIZAGI } \\
\text { FREE }\end{array}$ & $N A j$ & $\begin{array}{l}\text { BIZAGI } \\
\text { XPRESS }\end{array}$ & $N A j$ & $\begin{array}{l}\text { ARIS } \\
\text { FREE }\end{array}$ & $N A j$ & $\begin{array}{l}\text { ARIS } \\
\text { FULL }\end{array}$ & $N A j$ \\
\hline 1.2 & $\begin{array}{l}\text { Linguagem no idíoma nato (Português - } \\
\text { BR) }\end{array}$ & 2 & 1 & 2 & 1 & 2 & 3 & 6 & 1 & 2 & 3 & 6 & 3 & 6 & 1 & 2 & 3 & 6 \\
\hline 1.3 & Possui recurso Colaborativo & 2 & 3 & 6 & 3 & 6 & 3 & 6 & 1 & 2 & 1 & 2 & 3 & 6 & 2 & 4 & 3 & 6 \\
\hline 1.4 & Repositório integrado para os modelos & 2 & 3 & 6 & 3 & 6 & 3 & 6 & 1 & 2 & 1 & 2 & 3 & 6 & 1 & 2 & 3 & 6 \\
\hline 1.5 & Permite Versionamento & 2 & 1 & 2 & 3 & 6 & 3 & 6 & 1 & 2 & 1 & 2 & 1 & 2 & 1 & 2 & 3 & 6 \\
\hline 1.6 & Permite Publicação WEB & 2 & 3 & 6 & 3 & 6 & 3 & 6 & 3 & 6 & 3 & 6 & 3 & 6 & 1 & 2 & 3 & 6 \\
\hline 1.7 & $\begin{array}{l}\text { Permite exportação para outras } \\
\text { ferramentas através de XPDL. }\end{array}$ & 2 & 2 & 4 & 3 & 6 & 3 & 6 & 1 & 2 & 3 & 6 & 3 & 6 & 1 & 2 & 2 & 4 \\
\hline 1.8 & $\begin{array}{l}\text { Permite integração com outras } \\
\text { ferramentas de Workflow via BPEL }\end{array}$ & 4 & 2 & 8 & 1 & 4 & 3 & 12 & 1 & 4 & 3 & 12 & 3 & 12 & 1 & 4 & 3 & 12 \\
\hline 1.9 & Permite validação para o método & 2 & 2 & 4 & 3 & 6 & 3 & 6 & 1 & 2 & 3 & 6 & 3 & 6 & 3 & 6 & 3 & 6 \\
\hline 1.10 & $\begin{array}{l}\text { Permite consulta ao banco de dados por } \\
\text { entidade ontológica }\end{array}$ & 2 & 3 & 6 & 3 & 6 & 3 & 6 & 1 & 2 & 1 & 2 & 2 & 4 & 1 & 2 & 3 & 6 \\
\hline 1.11 & $\begin{array}{l}\text { Navegação entre modelos de diferentes } \\
\text { níveis }\end{array}$ & 2 & 2 & 4 & 2 & 4 & 2 & 4 & 1 & 2 & 1 & 2 & 2 & 4 & 1 & 2 & 3 & 6 \\
\hline 1.12 & $\begin{array}{l}\text { Atributos adicionais para os objetos do } \\
\text { modelo }\end{array}$ & 2 & 3 & 6 & 3 & 6 & 3 & 6 & 3 & 6 & 3 & 6 & 3 & 6 & 3 & 6 & 3 & 6 \\
\hline 1.13 & $\begin{array}{l}\text { Permite a utilização de mais de uma } \\
\text { notação de modelagem }\end{array}$ & 2 & 1 & 2 & 1 & 2 & 1 & 2 & 1 & 2 & 1 & 2 & 1 & 2 & 3 & 6 & 3 & 6 \\
\hline 1.14 & $\begin{array}{l}\text { Permite criar novos objetos } \\
\text { personalizados }\end{array}$ & 2 & 2 & 4 & 2 & 4 & 3 & 6 & 2 & 4 & 1 & 2 & 2 & 4 & 1 & 2 & 3 & 6 \\
\hline 1.15 & $\begin{array}{l}\text { Permite adicionar hyperlinks para outras } \\
\text { documentações }\end{array}$ & 2 & 2 & 4 & 2 & 4 & 2 & 4 & 1 & 2 & 2 & 4 & 3 & 6 & 3 & 6 & 3 & 6 \\
\hline \multirow[t]{2}{*}{1.16} & $\begin{array}{l}\text { Permite criar filtro com o conjunto de } \\
\text { objetos que se deseja usar }\end{array}$ & 2 & 2 & 4 & 2 & 0 & 2 & 4 & 1 & 2 & 1 & 2 & 2 & 4 & 1 & 2 & 3 & 6 \\
\hline & Média ponderada de requisitos & & & 4,53 & & 4,80 & & 5,73 & & $\mathbf{2 , 8 0}$ & & 4,13 & & 5,33 & & 3,33 & & 6,27 \\
\hline 2 & Suporte adicional & PESO & MEGA & $N A j$ & TIBCO & $N A j$ & $Q P R$ & $N A j$ & $\begin{array}{c}\text { QPR } \\
\text { EXPRESS }\end{array}$ & $N A j$ & $\begin{array}{l}\text { BIZAGI } \\
\text { FREE }\end{array}$ & $N A j$ & $\begin{array}{l}\text { BIZAGI } \\
\text { XPRESS }\end{array}$ & $N A j$ & $\begin{array}{l}\text { ARIS } \\
\text { FREE }\end{array}$ & $N A j$ & $\begin{array}{l}\text { ARIS } \\
\text { FULL }\end{array}$ & $N A j$ \\
\hline 2.1 & Existem cursos para treinamento & 1 & 2 & 2 & 2 & 2 & 3 & 3 & 1 & 1 & 3 & 3 & 3 & 3 & 3 & 3 & 3 & 3 \\
\hline 2.2 & Existem comunidades virtuais de apoio & 1 & 1 & 1 & 2 & 2 & 3 & 3 & 3 & 3 & 3 & 3 & 3 & 3 & 2 & 2 & 2 & 2 \\
\hline \multirow[t]{3}{*}{2.3} & $\begin{array}{l}\text { Existem publicações de apoio e suporte } \\
\text { disponíveis }\end{array}$ & 1 & 2 & 2 & 2 & 2 & 3 & 3 & 3 & 3 & 3 & 3 & 3 & 3 & 3 & 3 & 3 & 3 \\
\hline & Média ponderada de suporte adicional & & & 1,67 & & 2,00 & & 3,00 & & 2,33 & & 3,00 & & 3,00 & & 2,67 & & 2,67 \\
\hline & Média Final & & & 3,10 & & $\mathbf{3 , 4 0}$ & & 4,37 & & 2,57 & & 3,57 & & 4,17 & & 3,00 & & 4,47 \\
\hline
\end{tabular}




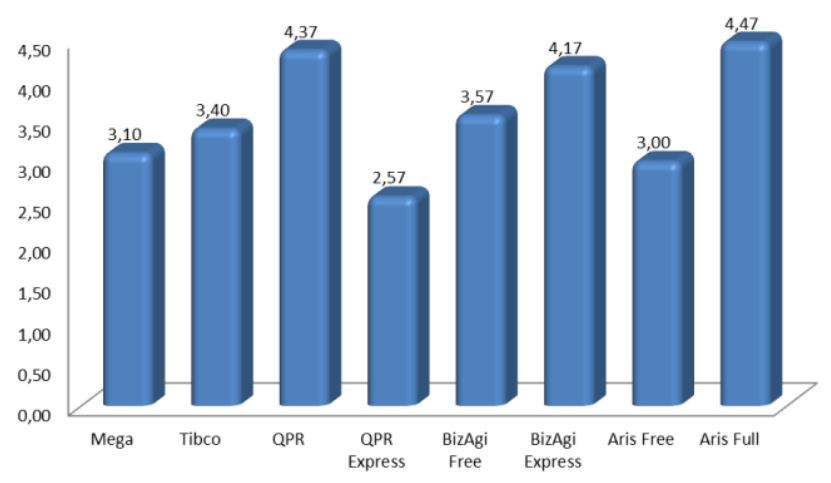

Figura 5. Média final comparativa entre ferramentas.

\section{Conclusão}

Após a realização de modelagem e análise por um grupo de especialistas, seguido de uma análise por usuários comuns, a pesquisa aponta para a notação BPMN como solução mais compreensível por humanos e com potencial para ser traduzida para máquinas. Ainda, sobre ferramentas, ficam indicadas a ARIS completa, a QPR Express, e a BizAgi, esta última gratuita.

Uma importante contribuição do trabalho foi considerar notações e ferramentas cuja relação foi ainda pouco explorada em trabalhos anteriores. Ainda, foram consideradas apenas ferramentas específicas de modelagem de processos. Outra contribuição foi a estruturação de critérios a partir de trabalhos anteriores, e ainda a adição de critérios específicos aos objetivos abordados pela pesquisa. Por fim, a recomendação de um método e de uma ferramenta específicos, oferece mais concretude e base para o avanço da pesquisa nessa área.

\section{Referências}

ABDALA, M.; LAHOZ, C.; SANT'ANNA, N. Utilizando o SPEM para a modelagem dos processos da qualidade e do gerenciamento da configuração em um ambiente integrado. V Simpósio Internacial de Melhora de Processo de Software, 2003.

AGULAR-SAVÉN, R. S. Business process modelling: review and framework. International Journal of Production Economics, 2003.

ALEXANDRE, J. W. C. Análise do número de categorias da escala Likert aplicada a gestão pela qualidade total através da teoria da resposta ao item. Encontro Nacional de Engehnaria de PRodução, 2003.

ARAUJO, R. et al. A definição de processos de software sob o ponto de vista da gestão de processos de negócio. IV Simpósio Internacional de Melhoria de Processos de Software, São Paulo, 2004.

BARGIS, J. The importance of business process modeling in software system design. Sicence of Computer Programming, 2008.

BENEDICTIS, C.; AMARAL, D. C.; ROZENDFELD, H. Avaliação dos principais métodos e ferramentas disponíveis para a modelagem do processo de desenvolvimento 
de produto. IV Congresso Brasileiro de Gestão de Desenvolvimento de Produtos, 2003.

GARTNER GROUP. Magic Quadrant for Business Process Analysis Tools. [S.1.]. 2008. (ID: G00161090).

GONZALES-PEREZ, C.; HENDERSON-SELLERS, B. Modelling software development methodologies: a conceptual foundation. The journal of Systems and Software, 2007.

GREEN, P.; ROSEMANN, M. Integrated process modeling: an ontological evaluation. Information Systems, 25, n. 2, 2000.

IEEE. Standard for Functional Modeling Language Syntax and Semantics for IDEF0. IEEE Explore, 1998.

MACEDO, R. D. S.; SCHMITZ, E. A. Ferramentas de modelagem de processo: uma avaliação. XXXIII Simpósio Brasileiro de Pesquisa Operacional (SBPO), 2001.

NORTON, D.; BLECHAR, M.; JONES, T. Magic quadrant for business process analysis tools. [S.1.]. 2010.

OMG. Business Process Model and Notation (BPMN). [S.1.]. 2010. (OMG DOcumento Number: dtc/2010-06-05).

ORTIZ-HERNÁNDEZ, J. et al. SOQUA'07. Dubrovnik, Coratia: [s.n.]. 2007.

RECKER, C. J. Opportuniites and constraints: the current struggle witdh BPMN. Business Process Management Journal, 2010. 181-201.

SANTOS JR, P. S.; ALMEIDA, J. P. A.; GUIZZARDI, G. An ontology-based semantic foundation for ARIS EPCs. ACM Symposium on applied computing, 2010.

SANTOS JR, P. S.; ALMEIDA, J. P.; PIANISSOLLA, T. L. Construção de um metamodelo para ARIS Method: escavação, refatoração e análise. Third Workshop on ontologies and metamodeling in software and data engeneering, 2008.

SCHEER, A.-W. ARIS Toolset: A Software product is born. ScienceDirect, 2003.

SOUNG-HIE, K.; KI-JIN, J. Desiging performance analysis and IDEF0 for enterprise modelling BPR. Elsevier International Journalof Production Economics, 2000.

SOUNG-HIE, K.; KI-JIN, J. Designing performance analysis and IDEF0 for enterprise modelling in BPR. International journal of production economics, 2002.

SOUZA, J. et al. Integração semântica de modelos. Rio de Janeiro. 2009. $(0001 / 2009)$.

WHITE, S. Introduction do BPMN. BPTrends, 2004. 